ISSN 0105-8517

\title{
On the Stability of ADI Methods
}

\section{Ole Østerby}

DAIMI PB - 598

April 2016

DEPARTMENT OF COMPUTER SCIENCE AARHUS UNIVERSITY

IT-parken, Aabogade 34 DK-8200 Aarhus N, Denmark

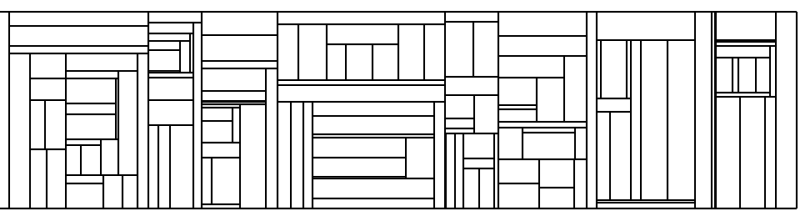




\title{
On the stability of ADI methods
}

\author{
Ole Østerby \\ Department of Computer Science \\ Aarhus University, Denmark. \\ oleby@cs.au.dk
}

\begin{abstract}
When solving parabolic equations in two space dimensions implicit methods are preferred to the explicit method because of their better stability properties. Straightforward implementation of implicit methods require time-consuming solution of large systems of linear equations, and ADI methods are preferred instead. We expect the ADI methods to inherit the stability properties of the implicit methods they are derived from, and we demonstrate that this is partly true. The Douglas-Rachford and Peaceman-Rachford methods are absolutely stable in the sense that their growth factors are $\leq 1$ in absolute value. Near jump discontinuities, however, there are differences w.r.t. how the ADI methods react to the situation: do they produce oscillations and how effectively do they damp them. We demonstrate the behaviour on two simple examples.
\end{abstract}

Keywords: ADI, stability, growth factors, oscillations.

MSC 65M06, 65M12

\section{Introduction}

In this paper we study various finite difference methods for solving parabolic equations in two space dimensions:

$$
u_{t}=P_{1} u+P_{2} u
$$

where $P_{1}$ and $P_{2}$ are differential operators involving partial derivatives of orders 0,1 , and 2 in $x$ and $y$, respectively. As a simple example we shall use $P_{1} u=b_{1} u_{x x}$ and $P_{2} u=b_{2} u_{y y}$ such that the equation is

$$
u_{t}=b_{1} u_{x x}+b_{2} u_{y y}
$$

The explicit method (EX) for solving (1) is

$$
\Delta_{t} v^{n}=P_{1 h}^{n} v^{n}+P_{2 h}^{n} v^{n}
$$

where $P_{1 h}$ and $P_{2 h}$ are finite difference operators approximating $P_{1}$ and $P_{2}$, respectively, and $v$ is the finite difference approximation to the true solution $u$. In the case of (2) we have

$$
\frac{v_{l m}^{n+1}-v_{l m}^{n}}{k}=b_{1} \frac{v_{l+1, m}^{n}-2 v_{l, m}^{n}+v_{l-1, m}^{n}}{h_{1}^{2}}+b_{2} \frac{v_{l, m+1}^{n}-2 v_{l, m}^{n}+v_{l, m-1}^{n}}{h_{2}^{2}}
$$


where $h_{1}, h_{2}$, and $k$ are the step sizes in the $x-, y$-, and $t$-direction, respectively, and $l, m$, and $n$ are the corresponding step numbers. Here and in the following we shall use the notation of [8] (see pp. 6ff and pp. 103ff).

To study the stability we use the von Neumann approach ([1], [8], p. 23) and compute the growth factor

$$
g_{E X}(\varphi)=1-4 b_{1} \mu_{1} \sin ^{2} \frac{\varphi_{1}}{2}-4 b_{2} \mu_{2} \sin ^{2} \frac{\varphi_{2}}{2}
$$

where $\mu_{1}=k / h_{1}^{2}$ and $\mu_{2}=k / h_{2}^{2}$, and $-\pi \leq \varphi_{1}, \varphi_{2} \leq \pi$. For stability we must have $|g(\varphi)| \leq 1$ for all $\varphi$ which puts severe restrictions on the time step size, in our case e.g.

$$
b_{i} \mu_{i} \leq \frac{1}{4} \quad \text { or } \quad k \leq \frac{h_{i}^{2}}{4 b_{i}}, \quad i=1,2
$$

We notice in passing that the critical cases occur for $\varphi_{i}$ close to $\pm \pi$. We call these solution components for high-frequency components because they correspond to solutions which oscillate between plus and minus at consecutive grid points (cf. [8], p. 21 and p. 57). Such components are dominant near a jump discontinuity, but are also introduced (with small amplitude) in continuous problems because of rounding errors. In these cases the condition $|g(\varphi)| \leq 1$ is (necessary and) sufficient to keep such components small. Near a jump discontinuity we would prefer the growth factor to be smaller in absolute value in order to damp out the annoying oscillations.

In the following we shall use the short-hand notation

$$
x_{i}=b_{i} \mu_{i} \sin ^{2} \frac{\varphi_{i}}{2}, \quad i=1,2,
$$

and note that $0 \leq x_{i} \leq b_{i} \mu_{i}$, the maximum value to be attained for high frequency components.

To avoid the step size restrictions we might prefer the implicit method (IM) [5]:

$$
\Delta_{t} v^{n}=P_{1 h}^{n+1} v^{n+1}+P_{2 h}^{n+1} v^{n+1}
$$

whose growth factor

$$
g_{I M}(\varphi)=\frac{1}{1+4 x_{1}+4 x_{2}}
$$

satisfies $0 \leq g(\varphi) \leq 1$ implying absolute stability. To advance the solution one time step we must now solve a system of linear equations which is rather timeconsuming. Instead we would prefer to use the Traditional Douglas-Rachford (TDR) ADI-method [3]:

$$
\begin{array}{ll}
\left(I-k P_{1 h}^{n+1}\right) \tilde{v} & =\left(I+k P_{2 h}^{n}\right) v^{n} \\
\left(I-k P_{2 h}^{n+1}\right) v^{n+1} & =\tilde{v}-k P_{2 h}^{n} v^{n}
\end{array}
$$


which involves solving two tridiagonal systems of equations per time step. In the derivation of TDR ([8], p. 112) it appears that it is unnecessarily complicated. Another possibility which we shall call the Simple Douglas-Rachford (SDR) method has the same error order in time $(=1)$ and is written

$$
\begin{aligned}
& \left(I-k P_{1 h}^{n+1}\right) \tilde{v}=v^{n} \\
& \left(I-k P_{2 h}^{n+1}\right) v^{n+1}=\tilde{v} .
\end{aligned}
$$

The growth factor for TDR is ([8], p. 113)

$$
g_{T D R}(\varphi)=\frac{1+16 x_{1} x_{2}}{1+4 x_{1}+4 x_{2}+16 x_{1} x_{2}}
$$

and for SDR

$$
g_{S D R}(\varphi)=\frac{1}{1+4 x_{1}+4 x_{2}+16 x_{1} x_{2}}
$$

The above-mentioned methods are only first order accurate in time. A second order method is the two-dimensional Crank-Nicolson (CN) method [2]:

$$
\Delta_{t} v^{n}=\frac{1}{2}\left(P_{1 h}^{n+1} v^{n+1}+P_{2 h}^{n+1} v^{n+1}\right)+\frac{1}{2}\left(P_{1 h}^{n} v^{n}+P_{2 h}^{n} v^{n}\right)
$$

The growth factor is

$$
g_{C N}(\varphi)=\frac{1-2 x_{1}-2 x_{2}}{1+2 x_{1}+2 x_{2}}
$$

and satisfies $-1 \leq g(\varphi) \leq 1$ again implying absolute stability.

To save computer time we again might prefer an ADI method, in this case Peaceman-Rachford (PR) [6]

$$
\begin{aligned}
& \left(I-\frac{1}{2} k P_{1 h}^{n+1}\right) \tilde{v} \quad=\left(I+\frac{1}{2} k P_{2 h}^{n}\right) v^{n}, \\
& \left(I-\frac{1}{2} k P_{2 h}^{n+1}\right) v^{n+1}=\left(I+\frac{1}{2} k P_{1 h}^{n}\right) \tilde{v}
\end{aligned}
$$

with growth factor $([8]$, p. 110)

$$
g_{P R}(\varphi)=\frac{\left(1-2 x_{1}\right)\left(1-2 x_{2}\right)}{\left(1+2 x_{1}\right)\left(1+2 x_{2}\right)} .
$$

\section{$2 \quad$ Stability and damping}

In continuous problems high frequency components are introduced by rounding errors. They therefore have small amplitudes, and the requirement $|g(\varphi)| \leq 1$ is 
perfectly satisfactory for keeping the amplitudes small. This is the case for all the above mentioned methods (with the exception of $\mathbf{E X}$ ).

In problems involving a jump discontinuity the high frequency components have large amplitudes (cf. [8], p. 21). These components are effectively damped in the true solution (cf. [8], p. 23), and we would wish the same to be true for the numerical solution. Therefore we should like $g(\varphi)$ to be small for $\varphi$ close to $\pi$.

Looking at the growth factor for $\operatorname{IM}(3)$ we note that $g_{I M}(\varphi)$ is small when $x_{1}$ and or $x_{2}$ is large, signalling that high frequency components are effectively damped. The same is true for SDR, but not for TDR where $g_{T D R}(\varphi)$ approaches 1 when both $x_{1}$ and $x_{2}$ are large.

The growth factor for $\mathbf{C N}$ approaches -1 when $x_{1}$ and/or $x_{2}$ is large. This means that high frequency components (which oscillate in $x$ or $y$ ) will also oscillate in $t$, the well-known $\mathbf{C N}$-oscillations. These oscillations are annoying but nevertheless give fair warning that the time step size is too large [4] and that certain measures should be taken to restore the physical significance of the numerical solution [7]. Looking at the growth factor for PR (7) we note that when $x_{1}$ or $x_{2}$ is large we have a similar situation with $g_{P R}(\varphi)$ approaching -1 , but when both $x_{1}$ and $x_{2}$ are large then $g_{P R}(\varphi) \approx 1$ such that (as with TDR) high frequency components are slowly damped but with no 'wiggles' in time to reveal that fact.

\section{$3 \quad$ Two test examples}

To investigate in practice the properties of the above methods we study two examples based on equation (2) with $b_{1}=b_{2}=1$ on the unit square with a jump discontinuity in the initial condition:

$$
\text { Example 1: } u(0, x, y)=\left\{\begin{array}{cc}
1 & x<y \\
0 & x=y \\
-1 & x>y
\end{array}\right.
$$

The boundary conditions for $t>0$ are derived from the true solution:

$$
\begin{gathered}
u(t, x, y)=\frac{4}{\pi} \sum_{j=0}^{\infty} \frac{1}{2 j+1} e^{-\frac{1}{2} \pi^{2}(2 j+1)^{2} t} \sin \left((2 j+1) \frac{\pi}{2}(x-y+2)\right) \\
\text { Example 2: } \\
u(0, x, y)=\left\{\begin{array}{cl}
1 & x<0.5 \\
0 & x=0.5 \\
-1 & x>0.5
\end{array}\right.
\end{gathered}
$$

with the true solution

$$
u(t, x, y)=\frac{4}{\pi} \sum_{j=0}^{\infty} \frac{1}{2 j+1} e^{-4 \pi^{2}(2 j+1)^{2} t} \sin ((2 j+1) 2 \pi x) .
$$


The step sizes in the $x-, y$-, and $t$-direction are $h_{1}=h_{2}=k=0.025$ such that $\mu_{1}=\mu_{2}=40$. In example 1 we have high-frequency solution components in both the $x$ - and the $y$-direction at $t=0$ and we therefore expect values of both $x_{1}$ and $x_{2}$ close to 40 . In example 2 we only have a jump discontinuity when travelling in the $x$-direction and therefore only expect $x_{1}$ to be close to 40 , while $x_{2}$ is small since any high frequency components in the $y$-direction will have very small amplitudes.

\section{Results}

Rather than presenting the complete two-dimensional solutions at each time step we have selected (typical) examples and show the solutions at specific lines close to the location of the jump. The results are shown in the following 6 figures. Each figure contains 6 curves, labeled true, IM, TDR, SDR, CN, and PR. Fig. 1-3 correspond to example 1 and Fig. 4-6 to example 2.

Fig. 1 and 4 show the $x$-dependence of $u(t, x, y)$ at the first time step, $t=0.025$, for $y=0.5$ and $0 \leq x \leq 1$,

Fig. 2 and 5 show $u(t, x, y)$ at the second time step, $t=0.05$, for the same values of $y$ and $x$, and

Fig. 3 and 6 show the $t$-dependence of $u(t, x, y)$ one $x$-step away from the jump at $x=0.525, y=0.5$, and $0 \leq t \leq 1$.

The findings confirm the predictions which can be made from the expressions for the growth factors.

In Example 1 where $x_{1} \approx x_{2} \approx 40$ both IM and SDR perform well (with SDR slightly better) whereas TDR shows a high frequency component (Fig. 1 and 2) which is very weakly damped $\left(g \approx \frac{25601}{25921} \approx 0.988\right.$ ) (cf. Fig. 3$)$. CN also shows a high frequency component which is damped with a negative $g\left(\approx-\frac{159}{161} \approx-0.988\right)$ giving rise to the well-known wiggles in time (cf. Fig. 3) whereas $\mathbf{P R}$ has a positive $g\left(\approx\left(\frac{-79}{81}\right)^{2} \approx 0.951\right)$ and has monotone behaviour in $t$. SDR is the winner with IM a close runner-up.

In Example 2 where $x_{1} \approx 40$ and $x_{2}$ is small IM, TDR, and SDR perform equally well showing good damping of the high frequency components $\left(g \approx \frac{1}{161} \approx 0.006\right)$. CN and PR both exhibit weakly damped oscillations due to negative growth factors. For $\mathbf{C N} g \approx \frac{-79}{81} \approx-0.975$ and for $\mathbf{P R}$ slightly better, since the smallest value for $\varphi_{2}$ is not 0 but $\frac{\pi}{40}$ (cf. [8], p. 56) such that $x_{2} \approx 40 \sin ^{2} \frac{\pi}{80} \approx 0.06$ and $g_{P R} \approx \frac{79}{81} \cdot \frac{0.88}{1.12} \approx 0.75$. After 10 steps the amplitude of the wiggles is reduced to $77 \%$ with CN and to $6 \%$ with PR (cf. Fig. 6 ). 


\section{References}

[1] G. G. O'Brien, M. A. Hyman, and S. Kaplan, A Study of the Numerical Solution of Partial Differential Equations, J. Math. Phys., 29 (1951), pp. 223-251. doi:10.1002/sapm1950291223

[2] J. Crank and P. Nicolson, A practical method for numerical evaluation of solutions of partial differential equations of the heat-conduction type, Proc. Cambridge Philos. Soc., 43 (1947), pp. 50-67.

Reprinted in Adv. Comput. Math., 6 (1996), pp. 207-226. doi:10.1007/BF02127704

[3] J. Douglas and H. H. Rachford, On the numerical solution of heat conduction problems in two and three space variables,

Trans. Amer. Math. Soc., 82 (1956), pp. 421-439.

doi:10.1090/S0002-9947-1956-0084194-4

[4] P. M. Gresho and R. L. Lee, Don't suppress the wiggles - they're telling you something, Computers and Fluids, 9 (1981), pp. 223-253.

doi:10.1016/0045-7930(81)90026-8

[5] P. Laasonen, Über eine Methode zur Lösung der Wärmeleitungsgleichung, Acta Math., 81 (1949), pp. 309-317.

[6] D. W. Peaceman and H. H. Rachford, The numerical solution of parabolic and elliptic differential equations, J. SIAM, 3 (1955), pp. 28-41. doi:10.1137/0103003

[7] O. Østerby, Five ways of reducing the Crank-Nicolson oscillations, BIT, 43 (2003), pp. 811-822. doi:10.1023/B:BITN.0000009942.00540.94

[8] O. Østerby, Numerical Solution of Parabolic Equations, Department of Computer Science, Aarhus University, 2015 doi:10.7146/aul.5.5. 


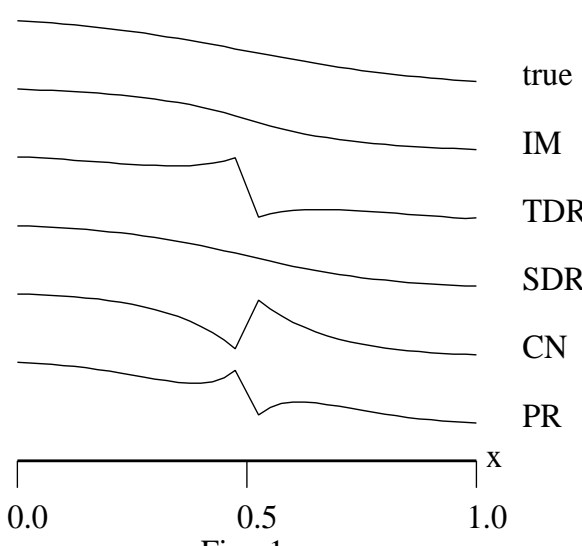

Fig. 1

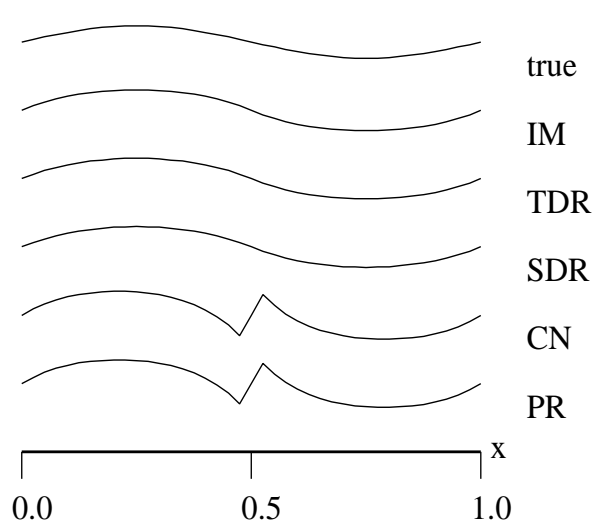

Fig. 4

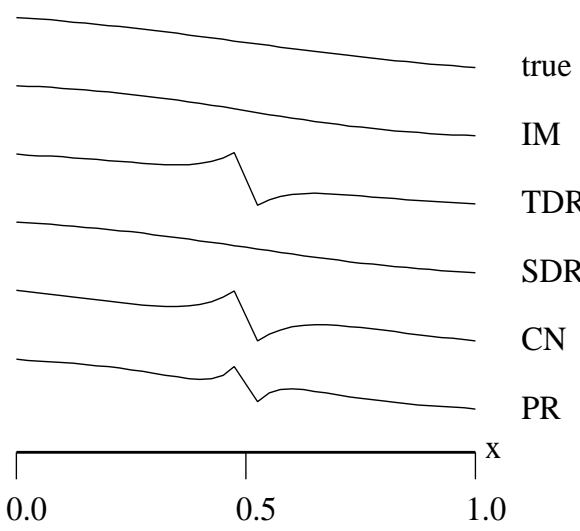

Fig. 2

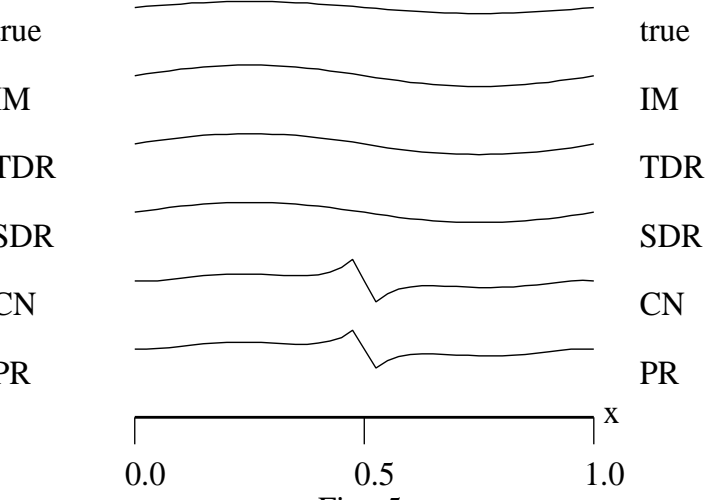

Fig. 5

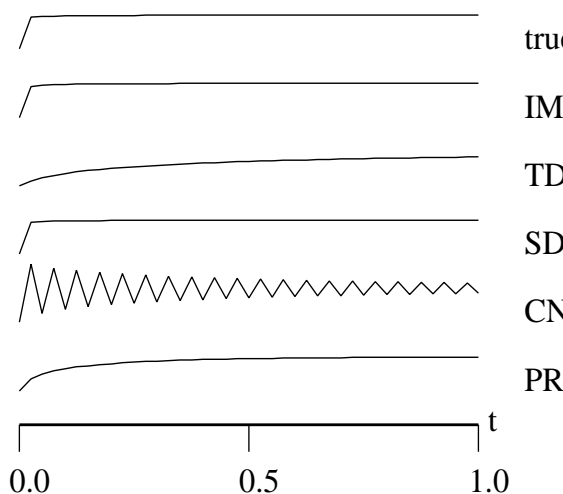

Fig. 3

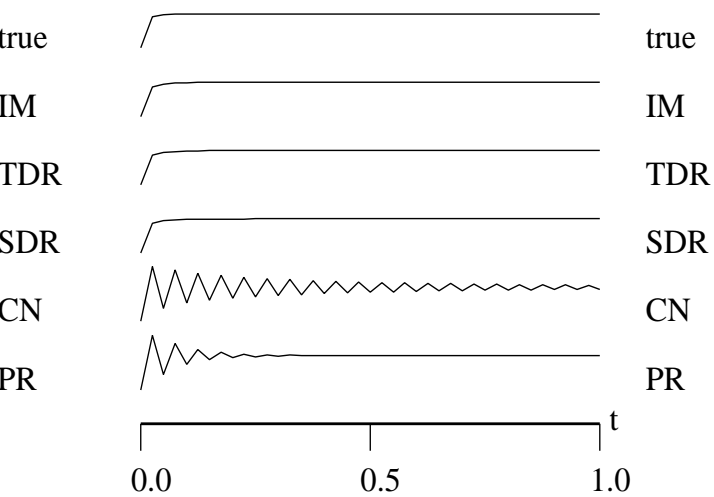

Fig. 6 